\title{
Nucleation and Initial Growth of Semiconductor Nanowires
}

\author{
Y. Cai*, S. K. Chan*, Y. F. Chan*, I. K. Sou*, D. S. Su** and N. Wang*
}

* Physics Department and the Institute of Nano Science and Technology, the Hong Kong University of Science and Technology, Hong Kong, China

** Department of Inorganic Chemistry, Fritz-Haber-Institute of the Max-Planck-Society, Faradayweg 4-6, D-14195 Berlin, Germany

The vapor-liquid-solid (VLS) [1] technique has been widely used for growing one-dimensional (1D) semiconductor nanostructures because the nucleation sites and the diameters of the 1D nanostructures can be well-controlled by the pre-formed metal catalysts. To better control the morphologies, atomic structures and defect densities of as-grown 1D semiconductor nanostructures, we have systematically investigated the formation mechanisms of semiconductor nanowires of different material systems (e.g. Si [2], III-V [3] and II-VI compounds [4]) by high-resolution transmission electron microscopy (HRTEM). We have observed that ultrathin semiconductor nanowires generally contained few defects and often exhibited unusual growth phenomena and unexpected properties, e.g. enhanced photothermal and photoacustic effects [5].

Here, as an example, we show our recent HRTEM study of nucleation and initial growth of II-VI compound semiconductor (e.g. ZnSe and $\mathrm{ZnS}$ ) nanowires. II-VI semiconductor nanowires were grown by molecular beam epitaxy technique via Au-catalyzed VLS reaction in this study (Fig.1). $\mathrm{ZnSe}$ (or $\mathrm{ZnS}$ ) nanowires with diameters greater than $30 \mathrm{~nm}$ always grow along $<111>$ direction on different surfaces of $\mathrm{GaAs}$ and $\mathrm{GaP}$ substrates. Those $\mathrm{ZnSe}$ nanowires having diameters of 10 $30 \mathrm{~nm}$ prefer $<112>$ growth on these substrates. Ultrathin ZnSe nanowires (diameters $<10 \mathrm{~nm}$ ) have only $<110>$ growth direction (Figs. 1 and 2). We have observed similar growth phenomena in various semiconductor nanowires (e.g. $\mathrm{Si}, \mathrm{GaAs}, \mathrm{ZnSe}$ and $\mathrm{ZnS}$ ) with a cubic structure. From our HRTEM and electron energy-loss spectroscopy studies, we found that Au catalysts initially reacted with these substrates forming binary $\mathrm{AuGa}_{2}$ alloy droplets (Fig.3 (a)-(e)). The sizes of the catalysts, the interfacial structures and the surface or interfacial energies determined the growth morphologies and directions of $\mathrm{ZnSe}$ nanowires.

The electrical transport properties of individual ZnSe nanowires (Fig.3 (f)-(g)) was studied in-situ by a nano-measurement system (a combination of HRTEM with the manipulation capacity of a scanning tunneling microscope). ZnSe nanowires showed n-type characteristics and their resistivities were measured to be about $1 \times 10^{2} \Omega \mathrm{cm}$. When applying a large current through a single crystalline $\mathrm{ZnSe}$ nanowire, we observed structural changes and material transportation through the nanowire.

\section{References}

[1] R. S. Wagner and W. C. Ellis, Appl. Phys. Lett. 4 (1964) 89.

[2] N. Wang, et al., Appl. Phys. Lett., 73 (1998)3902.

[3] W.S. Shi, et al., Adv. Mater. 13 (2001)591.

[4] Y. F. Chan, Appl. Phys. Lett. 83 (2003) 2665.

[5] N. Wang, et al., Nano Letters, 3 (2003) 475. 


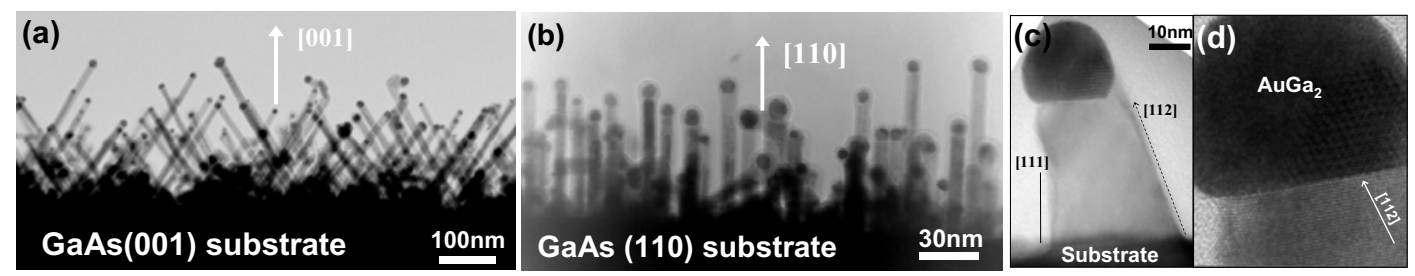

Fig. $1 \mathrm{ZnSe}$ nanowires (viewed in reflection mode of TEM) grown on (a) GaAs (001), (b) GaAs(110) and (c) GaAs (111) substrates. (c) and (d) Growth direction change of a $\mathrm{ZnSe}$ nanowire on a GaAs (111) substrate.
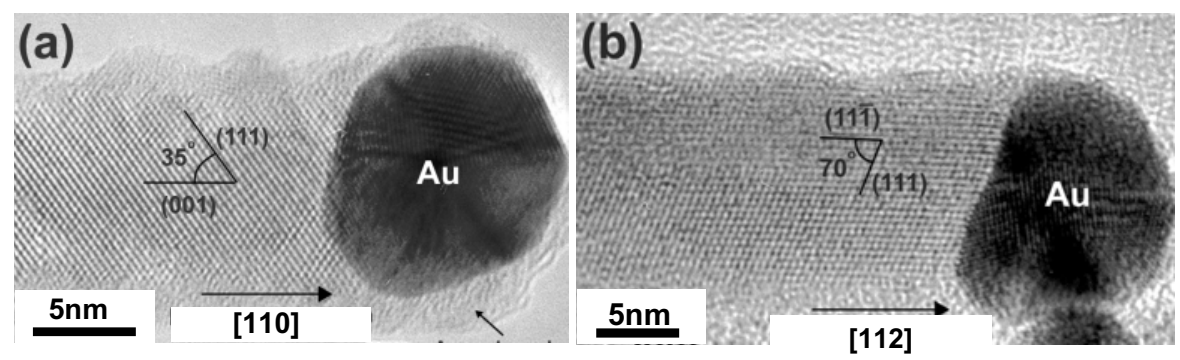

Fig. 2 HRTEM images of ZnSe nanowires grown along (a) [110] and (b) [112].
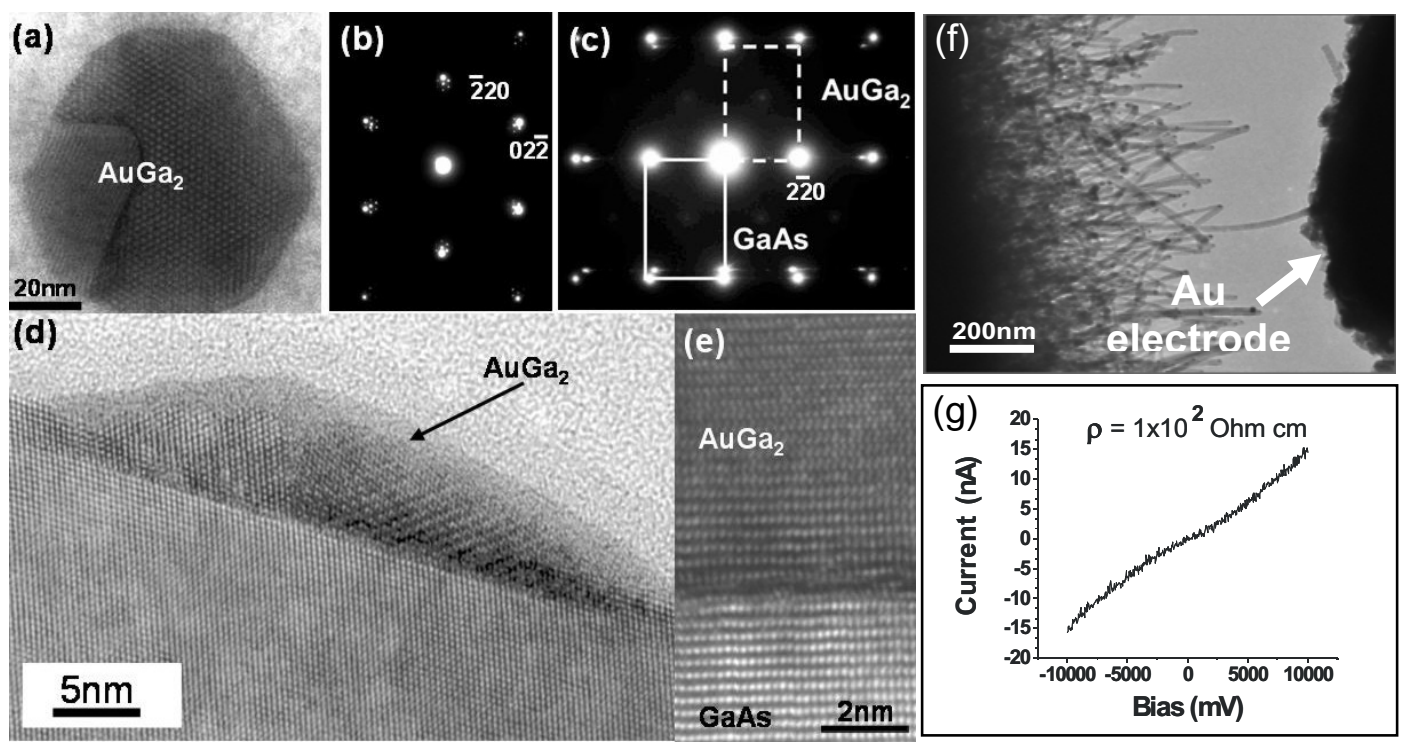

Fig. 3 (a) Plan-view of a AuGa 2 catalyst. (b) and (c) Electron diffraction patterns taken along the [111] and [112] zone axes of the catalyst respectively. (d) HRTEM cross-sectional view (along [110] direction) of an $\mathrm{AuGa}_{2}$ catalyst formed on GaAs substrate. (e) The catalyst interface structure viewed along [112]. (f) In-situ I-V measurement of a $\mathrm{ZnSe}$ nanowire. (g) I-V curve obtained from the nanowire shown in (f). 